\title{
Effects of oral adenosine-5'-triphosphate supplementation on athletic performance, skeletal muscle hypertrophy and recovery in resistance-trained men
}

Jacob M Wilson ${ }^{1 *}$, Jordan M Joy ${ }^{1}$, Ryan P Lowery ${ }^{1}$, Michael D Roberts ${ }^{2}$, Christopher M Lockwood ${ }^{3}$, Anssi H Manninen ${ }^{4}$, John C Fuller Jr. ${ }^{5}$, Eduardo O De Souza ${ }^{6}$, Shawn M Baier ${ }^{5}$, Stephanie MC Wilson ${ }^{7}$ and John A Rathmacher ${ }^{5,8}$

\begin{abstract}
Background: Currently, there is a lack of studies examining the effects of adenosine-5'-triphosphate (ATP) supplementation utilizing a long-term, periodized resistance-training program (RT) in resistance-trained populations. Therefore, we investigated the effects of 12 weeks of $400 \mathrm{mg}$ per day of oral ATP on muscular adaptations in trained individuals. We also sought to determine the effects of ATP on muscle protein breakdown, cortisol, and performance during an overreaching cycle.

Methods: The study was a 3-phase randomized, double-blind, and placebo- and diet-controlled intervention. Phase 1 was a periodized resistance-training program. Phase 2 consisted of a two week overreaching cycle in which volume and frequency were increased followed by a 2-week taper (Phase 3). Muscle mass, strength, and power were examined at weeks $0,4,8$, and 12 to assess the chronic effects of ATP; assessment performance variables also occurred at the end of weeks 9 and 10, corresponding to the mid and endpoints of the overreaching cycle.

Results: There were time $(p<0.001)$, and group $x$ time effects for increased total body strength $(+55.3 \pm 6.0 \mathrm{~kg}$ ATP vs. $+22.4 \pm 7.1 \mathrm{~kg}$ placebo, $p<0.001)$; increased vertical jump power (+ $796 \pm 75$ ATP vs. $614 \pm 52$ watts placebo, $p<0.001)$; and greater ultrasound determined muscle thickness ( $+4.9 \pm 1.0$ ATP vs. $(2.5 \pm 0.6 \mathrm{~mm}$ placebo, $p<0.02)$ with ATP supplementation. During the overreaching cycle, there were group $\mathrm{x}$ time effects for strength and power, which decreased to a greater extent in the placebo group. Protein breakdown was also lower in the ATP group.
\end{abstract}

Conclusions: Our results suggest oral ATP supplementation may enhance muscular adaptations following 12-weeks of resistance training, and prevent decrements in performance following overreaching. No statistically or clinically significant changes in blood chemistry or hematology were observed.

Trial registration: ClinicalTrials.gov NCT01508338

Keywords: Adenosine triphosphate, Exercise performance, Power, Strength, Muscle hypertrophy, Sports nutrition

\footnotetext{
* Correspondence: jmwilson@ut.edu

'Department of Health Sciences and Human Performance, The University of

Tampa, Tampa FL, USA

Full list of author information is available at the end of the article
} 


\section{Introduction}

Adenosine-5' -triphosphate's (ATP) role as the primary intracellular energy source for body tissues is well established [1]. In addition, ATP also has extensive extracellular functions that are primarily mediated through purinergic (P2Y and P2X) membrane receptors ubiquitously present in many cell types [2]. One extracellularmediated function of ATP includes the modification of muscle excitability (i.e., increasing skeletal muscle calcium permeability and blocking chloride efflux) and vasodilation [3,4]. Moreover as a co-transmitter, ATP operates on both the central and peripheral nervous systems to elicit local tissue modifications during neurotransmission [5].

It has been reported that the half-life of infused ATP is less than one second [6-8], ATP is rapidly taken up and stored by erythrocytes [6]. This rapid uptake by erythrocytes is central to its role in affecting blood flow and oxygen delivery to oxygen-depleted tissue [9]. Specifically, there is a tight coupling between oxygen demand in skeletal muscle and increases in blood flow. Erythrocytes regulate this response by acting as "oxygen sensors" [10]. When oxygen is low in a working muscle region, the red blood cell deforms, and releases ATP $[10,11]$. The result is vasodilation and greater blood flow to the working musculature, thereby enhancing nutrient and oxygen delivery $[10,11]$. Long-term oral administration of ATP has been shown to increase both the uptake and synthesis of ATP in the erythrocytes of rodents [12]. Collectively, these findings suggest that oral ATP supplementation may elicit ergogenic outcomes on skeletal muscle without elevating plasma ATP concentrations.

Research by Jordan et al. [13] demonstrated that $225 \mathrm{mg}$ per day of enteric-coated ATP supplementation for 15 days resulted in increased total bench press lifting volume (i.e. sets•repetitions•load $[\mathrm{kg}]$ ) as well as withingroup set-one repetitions to failure. More recently, Rathmacher et al. [14] found that 15 days of $400 \mathrm{mg}$ per day of ATP supplementation increased minimum peak torque for the final two sets of a dynamometer test. The increases suggest that orally delivered ATP may reduce muscle fatigue and enable a higher force output during repeated high-intensity bouts of exercise. These aforementioned findings lead us to hypothesize that dietary supplementation with ATP may be beneficial to both the exercising and less active muscle tissue.

The novelty of ATP as an oral supplement has limited data available in regards to its chronic effects in humans. To date, no studies have examined the chronic effects of oral ATP supplementation on body composition or indicators of athletic performance when combined with a periodized resistance training (RT) protocol. However, provided that short-term supplementation in the absence of a training intervention has resulted in positive outcomes in muscle performance, it is plausible to suggest that ATP may have long-term ergogenic effects in periodized RT regimens. Therefore, the primary purpose of this study was to test the hypothesis that supplementation with oral ATP would improve measures of power, strength, and skeletal muscle mass during a 12-week RT protocol. The second purpose was to assess the safety of the supplement over 12 weeks through blood chemistry and hematology measures.

\section{Methods}

\section{Study design}

This was a randomized, double-blind, placebo- and dietcontrolled, parallel groups with repeated measures study design. Both groups were assigned to a 12 -week periodized RT protocol. Blinding occurred via an outside researcher who sent the supplement and placebo in identical opaque capsules. This researcher was not involved in direct data collection, and did not meet any of the subjects. For this reason neither the researchers conducting the study nor the subjects knew who was in each group. Moreover the code was not broken until after all of the data were entered into Microsoft ${ }^{\circ}$ Excel, and sent to an outside researcher who was also blinded to the treatment groups. The protocol was divided into three phases. Phase one consisted of a three times per week non-linear periodized RT program for weeks $1-8$, modified from Kraemer et al. [15]. Phase two consisted of a two-week overreaching cycle during weeks 9 and 10. Finally, phase three consisted of participants tapering for weeks 11 and 12. Muscle mass and body composition were measured at baseline and at the end of weeks 4,8 , and 12. Muscle strength, vertical jump power, Wingate peak power (PP), creatine kinase (CK), C-reactive protein (CRP), free and total testosterone, and perceived recovery were measured at baseline and after weeks 4,8 , 9, 10 and 12. Additionally, CK, CRP, free and total testosterone, and perceived recovery were measured after week 1 of training. Protein breakdown was assessed using urinary 3-methylhistidine: Creatinine ratio (3-MH:Cr) at baseline and after weeks 1 and during the overreaching phase at weeks 8,9 , and 10 . These variables were used to assess the effects of ATP on performance, hormone status, and indices of muscle damage and recovery during an overreaching cycle. The ClinicalTrials.gov registration ID was NCT01508338.

\section{Participants}

Twenty-four resistance-trained males were selected for the study. However, due to injury three subjects dropped out of the study leaving 21 subjects (11 ATP supplemented and 10 placebo supplemented) aged 23.4 \pm 0.7 years, with an average one-repetition maximum (1RM) squat, bench press, and deadlift of $1.71 \pm 0.04$, $1.34 \pm 0.03$ and $2.05 \pm 0.04$ times their bodyweight were 
recruited for the study. Participants could not participate if they were taking an anti-inflammatory agent, a performance-enhancing supplement, if they smoked, or if they had consumed nutritional supplements during the three months prior to data collection. Each participant signed an informed consent approved by the University of Tampa Institutional Review Board before participating in the study.

\section{Muscle strength, power, body composition and skeletal muscle hypertrophy testing}

After familiarization procedures, muscle strength was assessed via 1RM testing of the back squat, bench press, and deadlift. Each lift was performed as described by the International Powerlifting Federation rules [16]. We used an intraclass correlation coefficient (ICC) $(2, \mathrm{k})$ formula [17] to determine the reliability of repeated measures within testers. The ICC for strength measures ranged from 0.96 to 0.98 . Body composition (lean body mass, fat mass, and total mass) was determined by dualenergy x-ray absorptiometry (DXA; Lunar Prodigy enCORE 2008, Madison, Wisconsin, U.S.A.). Skeletal muscle hypertrophy was determined via the combined changes in ultrasonography-determined muscle thickness of the vastus lateralis (VL) and vastus intermedius (VI) muscles. The mean of three measurements by the same blinded investigator were taken at $50 \%$ of femur length over the mid-belly of the muscle with the subjects lying in a supine position. The precision for the testretest of muscle thickness measurements was 0.975 .

Muscle power was assessed during maximal cycling and jumping movements. During the cycling test, volunteers were instructed to cycle against a predetermined resistance (7.5\% of body weight) as fast as possible for 10 seconds [18]. The saddle height was adjusted to the individual's height to produce a $5-10^{\circ}$ knee flexion while the foot was in the low position of the central void. A standardized verbal stimulus was provided to each participant. Power output was recorded in real time during the 10-second sprint test, by a computer connected to the standard cycle ergometer (Monark model 894e, Vansbro, Sweden). Peak power (PP) was recorded using Monark Anaerobic Wingate Software, Version 1.0 (Monark, Vansbro, Sweden). The ICC of muscle peak power was 0.96 .

Measurements of PP were also taken during a vertical jump (VJ) test performed on a multicomponent AMTI force platform (Advanced Mechanical Technology, Inc., Watertown, MA), interfaced with a personal computer at a sampling rate of $1000 \mathrm{~Hz}$ [19]. Data acquisition software (LabVIEW, version 7.1; National Instruments Corporation, Austin, TX) was used to calculate PP. Peak power was calculated as the peak combination of ground reaction force and peak velocity during the accelerated launch on the platform. The ICC of VJ power was 0.97 .

\section{Supplementation and diet control}

Prior to the study, participants were randomly assigned to receive either $400 \mathrm{mg}$ per day of ATP disodium or maltodextrin (placebo), consumed orally via a two-piece gelatin capsule 30 minutes prior to RT sessions. On non-training days, participants were instructed to consume one dose on an empty stomach prior to breakfast. The placebo and ATP capsules $(400 \mathrm{mg}$ ) were obtained directly from the commercial manufacturer (TSI USA Inc., Missoula, MT) and were produced in compliance with U.S. cGMPs for dietary supplements. The Certificate of Analysis for both the placebo and ATP capsules were provided and verified the supplement contents. Moreover, we verified the capsule's purity by HPLC. Two weeks prior to and throughout the study, participants were placed on a diet consisting of $25 \%$ protein, $50 \%$ carbohydrates, and $25 \%$ fat by a registered dietician who specialized in sport nutrition. Participants met as a group with the dietitian, and they were given individual meal plans two weeks prior to the onset of the study. Diet counseling was continued on an individual basis throughout the study.

\section{Resting blood draws}

All blood draws were obtained after an overnight 12-hour fast via venipuncture by a trained phlebotomist, and were scheduled at the same time of day to avoid influences of hormonal variations. Whole blood was collected and transferred into appropriate tubes and centrifuged at $1500 \mathrm{x} \mathrm{g}$ for $15 \mathrm{~min}$ at $4^{\circ} \mathrm{C}$. Resulting serum and plasma were then aliquoted and stored at $-80^{\circ} \mathrm{C}$ until subsequent analyses. A portion of the blood samples taken at weeks 0 , 4, 8, and 12 were used for measurements (ANY LAB TEST NOW ${ }^{\oplus}$, Tampa, Fl) of glucose, blood urea nitrogen, creatinine, eGFR, $\mathrm{Na}, \mathrm{K}, \mathrm{Cl}, \mathrm{CO}_{2}, \mathrm{Ca}$, protein, albumin, globulin, albumin:globulin ratio, total bilirubin, alkaline phosphatase, aspartate aminotransferase, and alanine aminotransferase. A complete blood count was also performed on each blood sample. The commercial laboratory performing the safety analysis was instructed to inform investigators of any abnormal values in any parameters measured during the study time points measured. In the case of an abnormal value, the subject would be told and not be allowed to continue the study. Because no abnormal values were detected during the study in these healthy and highly fit subjects, the total aggregate numbers were not presented to the investigators until the end of the study.

\section{Biochemical analysis}

Samples were thawed once and analyzed in duplicate for each analyte. Serum free and total testosterone, cortisol, 
and C-reactive protein (CRP) were assayed via ELISA kits obtained from Diagnostic Systems Laboratories (Webster, TX). All hormones were measured within the same assay and on the same day to avoid compounded inter-assay variance. Intra-assay variance was less than $3 \%$ for all analytes. Serum creatine kinase (CK) was measured using colorimetric procedures at $340 \mathrm{~nm}$ (Diagnostics Chemicals, Oxford, CT).

\section{Perceived recovery status scale}

Perceived Recovery Status (PRS) Scale was measured at all measurement times and in particular at weeks 8, 9, and 10 to assess participant recovery during the overreaching phase. The PRS Scale consists of values between $0-10$, with $0-2$ being very poorly recovered and characterized by anticipated declines in performance; 4-6 is defined as low-to-moderately recovered and characterized by no expected change in performance; and, 8-10 represents high perceived recovery and correlates strongly with increases in performance [20].

\section{Statistics}

A one-way ANOVA model was used to analyze the baseline characteristic data using the Proc GLM procedure in $\mathrm{SAS}^{\odot}$ (SAS Institute, Cary, NC). The main effect of treatment (Trt) was included in the model. Actual values for muscle strength and power, body composition, muscle damage, hormonal status, and PRS changes over the 12-week study were analyzed by using a repeated measures ANOVA using the Proc Mixed procedure in $\mathrm{SAS}^{\oplus}$. The initial baseline value (e.g. week 0 ) was used as a covariate with the main effects of Time, Trt, and the interaction Trt*Time in the model. The overreaching cycle of the study was further assessed by using repeated measures ANOVAs with the Proc Mixed procedure in $\mathrm{SAS}^{\oplus}$. Values measured at the week- 8 time point were used as a covariate with the main effects of Time, Trt, and Trt*Time for the overreaching phase (phase two). The Least Squares Means procedure was used to compare Trt means at each time point. Statistical significance was determined at $p \leq 0.05$.

\section{Results}

Participant characteristics

There were no differences in age (placebo $=23.0 \pm 1.2$, $\mathrm{ATP}=23.7 \pm 0.9 \mathrm{yrs})$, height $($ placebo $=180.6 \pm 2.3$, ATP $=$ $179.0 \pm 1.0 \mathrm{~cm}$ ), or body mass (placebo $=87.4 \pm 4.3$, ATP $=$ $85.7 \pm 1.7$ between the treatments at the start of the study.

Table 1 Effect of ATP supplementation on muscle strength and power in participants performing a 12 week weight training regimen. ${ }^{a}$

\begin{tabular}{|c|c|c|c|c|c|c|c|}
\hline & \multicolumn{6}{|c|}{ Week of Study } & \multirow[b]{2}{*}{$p$-value } \\
\hline & 0 & 4 & 8 & 9 & 10 & 12 & \\
\hline \multicolumn{8}{|l|}{ Squat, kg } \\
\hline Placebo & $145.2 \pm 10.2$ & $151.6 \pm 11.0$ & $156.4 \pm 11.6$ & $150.7 \pm 10.4$ & $147.2 \pm 10.7$ & $151.3 \pm 10.3$ & \\
\hline ATP & $146.1 \pm 5.8$ & $155.2 \pm 7.7$ & $160.7 \pm 7.6$ & $159.0 \pm 6.1^{\#}$ & $158.6 \pm 6.9^{\#}$ & $165.3 \pm 8.3^{\#}$ & $<0.001$ \\
\hline \multicolumn{8}{|c|}{ Bench Press, kg } \\
\hline Placebo & $115.2 \pm 9.4$ & $119.3 \pm 9.4$ & $121 . .4 \pm 9.3$ & $113.4 \pm 7.2$ & $115.7 \pm 8.0$ & $119.3 \pm 8.7$ & \\
\hline ATP & $117.2 \pm 5.5$ & $122.1 \pm 5.0$ & $124.5 \pm 5.2$ & $120.0 \pm 4.8$ & $121.2 \pm 5.5$ & $124.2 \pm 5.4$ & 0.65 \\
\hline \multicolumn{8}{|c|}{ Deadlift, kg } \\
\hline Placebo & $169.8 \pm 11.0$ & $177.0 \pm 10.7$ & $182.5 \pm 10.7$ & $173.2 \pm 10.8$ & $174.8 \pm 8.1$ & $182.0 \pm 9.2$ & \\
\hline ATP & $176.2 \pm 4.9$ & $189.2 \pm 5.9$ & $200.3 \pm 7.4^{\#}$ & $193.4 \pm 6.4^{\#}$ & $193.8 \pm 8.3^{\#}$ & $205.2 \pm 6.9^{\#}$ & 0.002 \\
\hline \multicolumn{8}{|c|}{ Total Strength, kg } \\
\hline Placebo & $430.2 \pm 28.1$ & $448.0 \pm 28.9$ & $460.2 \pm 29.6$ & $437.3 \pm 26.1$ & $437.6 \pm 25.1$ & $452.6 \pm 25.9$ & \\
\hline ATP & $439.4 \pm 14.9$ & $466.5 \pm 17.1$ & $485.6 \pm 18.1^{\#}$ & $472.4 \pm 15.2^{\#}$ & $473.6 \pm 18.3^{\#}$ & $494.7 \pm 17.9^{\#}$ & $<0.001$ \\
\hline \multicolumn{8}{|c|}{ Wingate Peak Power, Watts } \\
\hline Placebo & $882.2 \pm 54.1$ & $930.3 \pm 55.7$ & $985.3 \pm 64.7$ & $922.9 \pm 61.9$ & $939.9 \pm 65.7$ & $977.4 \pm 66.5$ & \\
\hline ATP & $882.8 \pm 27.0$ & $960.9 \pm 36.1$ & $1003.5 \pm 40.4$ & $964.2 \pm 38.0$ & $974.7 \pm 39.8$ & $1011.9 \pm 42.4$ & 0.48 \\
\hline \multicolumn{8}{|c|}{ Vertical Jump power, Watts } \\
\hline Placebo & $5318 \pm 410$ & $5711 \pm 439$ & $5918 \pm 443$ & $5697 \pm 436$ & $5626 \pm 431$ & $5932 \pm 452$ & \\
\hline ATP & $5185 \pm 203$ & $5618 \pm 213$ & $5954 \pm 202^{\#}$ & $5838 \pm 218^{\#}$ & $5827 \pm 208^{\#}$ & $5981 \pm 207^{\#}$ & $<0.001$ \\
\hline
\end{tabular}

${ }^{a}$ Mean \pm SEM for $n=11$ ATP (400 mg ATP/d taken 30 min prior to exercise) and $n=10$ placebo supplemented participants.

${ }^{b}$ Probability of treatment by time difference between the placebo and the ATP treatments over the 12-week study. The mixed model ANOVA in SAS ${ }^{\circledR}$ was used with the main effects of treatment, time and treatment by time, with the value for week 0 used as a covariate.

"Significantly different than corresponding placebo, $t$-test $(p<0.05)$. 


\section{Muscle strength and power}

Both groups increased their muscle strength (Table 1, Figure 1A, $t$-test, $p<0.001)$. However, supplementation with ATP resulted in significantly greater increases in the $1 \mathrm{RM}$ for the squat, deadlift, and total strength compared with placebo over the 12-week study (Trt"time, $p<0.001,0.002$, and 0.001 , respectively). The ATP supplementation resulted in strength increases of $12.9 \%$ and $16.4 \%$ for squat and deadlift, respectively. However, in the placebo group, $\mathrm{RT}$ alone resulted in increases of 4.4 and $8.5 \%$ for squat and deadlift, respectively. The total strength increases over the 12-week study were 5.9\% $(22.4 \pm 7.1 \mathrm{~kg})$ in the placebo-supplemented participants and $12.6 \%(55.3 \pm 6.0 \mathrm{~kg})$ in the ATP-supplemented participants (Figure 1A, Trt"time, $p<0.001$ ). Mean total strength in response to ATP supplementation was greater at $8,9,10$, and 12 weeks than mean total strength in the placebo-supplemented participants ( $t$-test, $p<0.05)$. During phase 2 , the overreaching cycle, the placebo-supplemented group had a $22.6 \pm 5.1 \mathrm{~kg}$ decrease in total strength, while the ATP-supplemented group decreased only $12.0 \pm 2.5 \mathrm{~kg}$ in total strength during the same test period (Table 1, Figure 1A, Trt, $p<0.007)$.

Muscle power measured by Wingate PP and vertical jump power increased pre-to-post-test similarly in both groups during the RT protocol (Table 1 and Figure 1B; $t$-test, $p<0.001)$. However, ATP supplementation significantly increased vertical jump power when compared to placebo (Table 1 and Figure 1B; Trt"time $p<0.001$ ). The ATP-supplemented participants had a $15.7 \%$ (796 \pm 75 watts) increase in vertical jump power compared with an $11.6 \%$ (614 \pm 52 watts) increase in placebo-supplemented participants over the 12-week period. During phase 2,
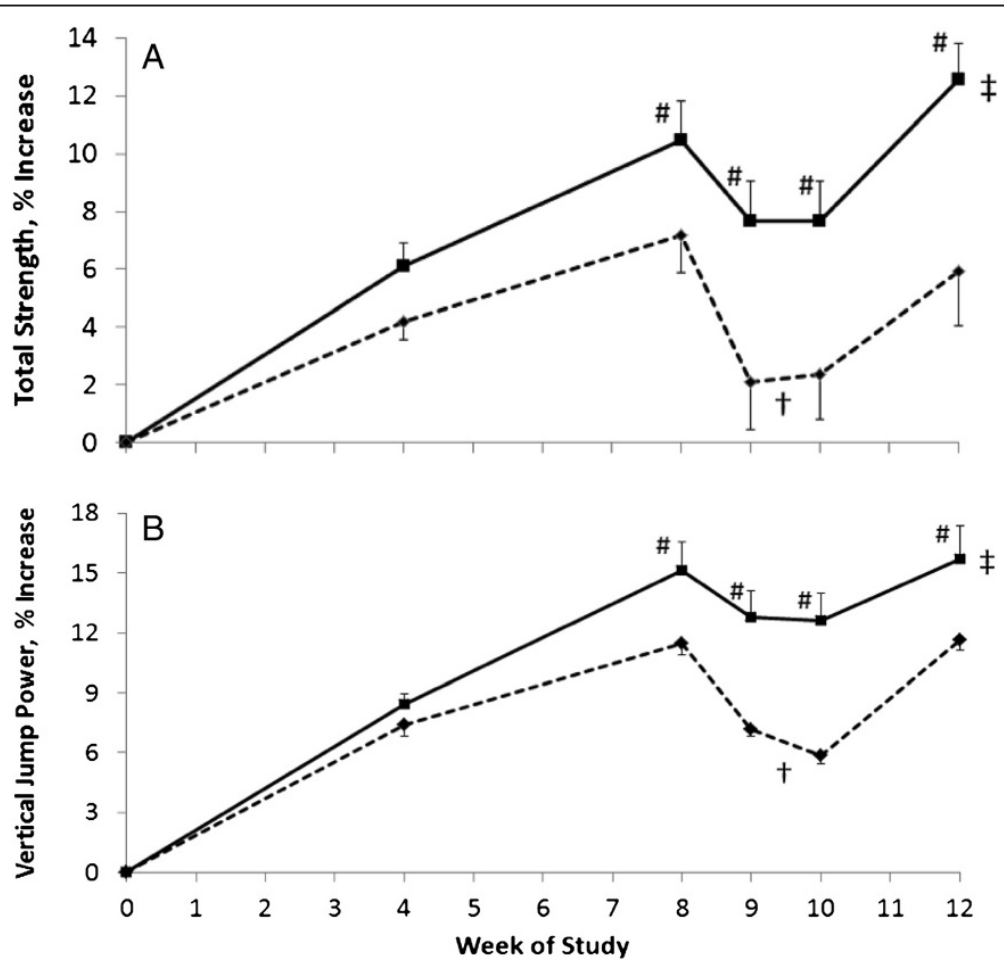

Figure 1 Effects of oral ATP supplementation on total strength and vertical jump power. (A) Percent change in total strength as the of sum of the 1-RM in bench press, squat, and deadlift in male participants undergoing 12 weeks of intense, periodized resistance training with an overreaching cycle during weeks 9 and 10. Participants were supplemented with either a Placebo - - - $(\mathrm{n}=10)$ or $400 \mathrm{mg}$ ATP/d - I- ( $\mathrm{n}=11)$ during the 12-week training program. When compared with placebo supplementation total strength was increased with ATP supplementation over the 12 week study ( $\neq p<0.001$, Trt*time). During the overreaching cycle of the study, total strength decreased in the placebosupplemented group relative to the ATP-supplemented group during weeks 9 and 10 ( $\uparrow p<0.001$, Trt*time). Additionally, participants supplemented with ATP had greater mean strength at the 8,9,10, and 12 week time-points compared with placebo-supplemented participants (\# $\mathrm{p}<0.05$ ). (B) Percent change in vertical jump power in male participants undergoing 12 weeks of intense, periodized resistance training with an overreaching cycle during weeks 9 and 10. Participants were supplemented with either a Placebo - - - $(\mathrm{n}=10)$ or $400 \mathrm{mg}$ ATP /d — I$(n=11)$ during the training program. ATP supplementation resulted in a greater increase in vertical jump power over the 12 week study ( $\neq p<0.001$, Trt*time). The increased intensity of the overreaching cycle during the study resulted in a significant decrease in vertical jump power in the placebo-supplemented participants compared with the ATP-supplemented participants during weeks 9 and 10 († $p<0.007)$. Additionally, ATP-supplemented participants had greater vertical jump power at 8, 9, 10, and 12 weeks of study compared with placebosupplemented participants (\# $p<0.05)$. 
the overreaching cycle, the placebo-supplemented participants had a $5.0 \%(-291 \pm 25$ watts $)$ decrease in power from week 8 to week 10, while the ATP-supplemented participants had a $2.2 \%$ decrease $(-126 \pm 19$ watts; Trt"time, $p<0.001$ ).

Body composition and muscle hypertrophy measurement After 12 weeks of RT, lean body mass (LBM) increased within both groups (Table 2 , $t$-test, $p<0.001$ ), whereas fat percentage was decreased with training only in the ATP-supplemented participants (Table $2 t$-test, $p<0.01$ ). The ATP supplementation did not affect overall body weight or fat percentage during the study, but it did significantly increase LBM gain during the 12-week training period (Trt*time, $p<0.009$ ). The ATP-supplemented participants gained $4.0 \pm 0.4 \mathrm{~kg}$ of LBM whereas the placebo-supplemented participants gained $2.1 \pm 0.5 \mathrm{~kg}$ of LBM. The quadriceps muscle thickness increased after 12 weeks of RT (Table 2, Time, $p<0.001$ ). Additionally, ATP supplementation resulted in a $9.4 \%(4.9 \pm 1.0 \mathrm{~mm})$ increase in quadriceps muscle thickness compared with a $4.9 \%(2.5 \pm 0.6 \mathrm{~mm})$ increase in response to placebo (Table 2, Trt*time $p<0.02$ ).

\section{Muscle damage, hormonal status and performance recovery scale}

Muscle damage assessed by blood CK was affected by training (Table 3 , Time, $p<0.001$ ). Supplementation with ATP was unable to attenuate the increase in CK at either

Table 2 Effect of ATP supplementation on body composition and Quadriceps thickness in participants performing a 12 week weight training regimen. ${ }^{a}$

\begin{tabular}{|c|c|c|c|c|c|}
\hline & \multicolumn{4}{|c|}{ Week of Study } & \multirow[b]{2}{*}{$p$-value ${ }^{b}$} \\
\hline & 0 & 4 & 8 & 12 & \\
\hline \multicolumn{6}{|c|}{ Weight, kg } \\
\hline Placebo & $87.4 \pm 4.3$ & $88.3 \pm 4.6$ & $88.7 \pm 4.8$ & $87.7 \pm 4.7$ & \\
\hline ATP & $85.7 \pm 1.7$ & $86.9 \pm 2.0$ & $87.0 \pm 2.0$ & $87.0 \pm 2.1$ & 0.49 \\
\hline \multicolumn{6}{|c|}{ DXA LBM, kg } \\
\hline Placebo & $68.5 \pm 2.6$ & $70.0 \pm 2.3$ & $71.2 \pm 2.4$ & $70.5 \pm 2.4$ & \\
\hline ATP & $67.7 \pm 2.0$ & $70.1 \pm 1.9$ & $71.4 \pm 2.0^{\#}$ & $71.7 \pm 1.9^{\#}$ & .009 \\
\hline \multicolumn{6}{|c|}{ DXA Fat,\% } \\
\hline Placebo & $21.0 \pm 1.1$ & $19.8 \pm 1.6$ & $18.6 \pm 1.9$ & $18.6 \pm 1.7$ & \\
\hline ATP & $19.5 \pm 1.8$ & $18.1 \pm 1.8$ & $16.6 \pm 1.6$ & $16.0 \pm 1.5$ & 0.76 \\
\hline \multicolumn{6}{|c|}{ Quad, mm } \\
\hline Placebo & $50.2 \pm 2.1$ & $52.2 \pm 2.3$ & $52.6 \pm 2.4$ & $52.7 \pm 2.4$ & \\
\hline ATP & $50.9 \pm 0.9$ & $53.4 \pm 1.3$ & $54.8 \pm 1.7$ & $55.8 \pm 1.8^{\#}$ & 0.02 \\
\hline
\end{tabular}

${ }^{a}$ Mean \pm SEM for $n=11$ ATP (400 mg ATP/d taken 30 min prior to exercise) and $n=10$ placebo supplemented participants.

${ }^{b}$ Probability of treatment by time difference between the placebo and the ATP treatments over the 12-week study. The mixed model ANOVA in SAS ${ }^{\circledR}$ was used with the main effects of treatment, time and treatment by time, with the value for week 0 used as a covariate.

${ }^{\#}$ Significantly different than corresponding placebo, $t$-test $(p<0.05)$. the initiation of training (weeks 0 to 1 ) or during phase 2 , the overreaching cycle (weeks 9 and 10), where the sudden change in training volumes caused blood CK to increase. Muscle protein degradation was measured by the urinary 3- $\mathrm{MH}: \mathrm{Cr}$ ratio (Table 3) during the initiation of training (weeks 0 to 1 ) and overreaching cycle (weeks 9 and 10). During week 8 of the study, ATP-supplemented subjects had a higher urinary 3-MH:Cr ratio compared to the placebo group ( $t$-test, $p<0.05)$. However, the 8-week values were not different than baseline for both treatment groups. The results indicate that when training volume increased during weeks 9 and 10, ATP supplementation significantly decreased muscle protein degradation compared with placebo (Trt*time, $p<0.007)$. During phase 2 , the overreaching cycle, protein degradation increased $23.7 \pm 4.5 \%$ in the placebosupplemented, but not ATP supplemented participants. Supplementation with ATP did not affect changes in CRP, cortisol, or free or total testosterone levels when compared with placebo supplementation (Table 3).

Muscle recovery and readiness to train in the next training session was measured by PRS score (Table 3). While no overall 12-week Trt*time effect of ATP supplementation was observed, the effects of ATP supplementation compared with placebo at weeks 10 and 12 may indicate that ATP supplementation improved perceived recovery (Table 3 ).

Safety assessment using blood chemistry and hematology Blood chemistry and hematology analyses were performed at baseline (week 0 ) and at weeks 4, 8, and 12 of the study; the data are shown in Tables 4 and 5, which displays blood chemistry and hematology values, respectively. No statistically or clinically significant changes in blood chemistry or hematology were observed over the 12-week ATP supplementation period when compared with placebo supplementation. There were no adverse events reported in this study.

\section{Discussion}

The primary findings of this study were that individuals consuming $400 \mathrm{mg}$ of oral ATP daily demonstrated ergogenic effects with ATP supplementation, since they showed greater gains in muscle mass, lean body mass, strength and power when compared to a placebomatched control.

The effects of ATP on skeletal muscle strength and power development

Strength and power are two of the most critical attributes underlying success in athletics [21,22]. These variables are intimately related and allow athletes to be successful in their respective sport $[23,24]$. Prior to our research, there was limited data evaluating the effects of 
Table 3 Blood creatine kinase (CK), urinary 3-methylhistidine (3MH), C-reactive protein (CRP), cortisol, free and total testosterone, and perceived recovery score (PRS) in participants performing a 12 week weight training regimen and supplemented with either a placebo or ATP. ${ }^{\text {a }}$

\begin{tabular}{|c|c|c|c|c|c|c|c|c|}
\hline & \multirow[b]{2}{*}{0} & \multicolumn{6}{|c|}{ Week of Study } & \multirow[b]{2}{*}{$p$-value } \\
\hline & & 1 & 4 & 8 & 9 & 10 & 12 & \\
\hline \multicolumn{9}{|l|}{$C K, I U / L$} \\
\hline Placebo & $141 \pm 12$ & $582 \pm 77$ & $373 \pm 13$ & $246 \pm 29$ & $484 \pm 52$ & $528 \pm 72$ & $187 \pm 21$ & \\
\hline ATP & $145 \pm 8$ & $500 \pm 71$ & $324 \pm 14$ & $234 \pm 32$ & $426 \pm 44$ & $449 \pm 62$ & $160 \pm 20$ & 0.91 \\
\hline \multicolumn{9}{|c|}{$24 \mathrm{~h} 3 \mathrm{MH}: \mathrm{Cr}, \mu \mathrm{mol}: \mathrm{mg}$} \\
\hline Placebo & $0.127 \pm 0.007$ & $0.130 \pm 0.003$ & $\mathrm{Nm}^{\mathrm{c}}$ & $0.123 \pm 0.004$ & $0.134 \pm 0.005$ & $0.152 \pm 0.005$ & $\mathrm{Nm}$ & \\
\hline ATP & $0.136 \pm 0.008$ & $0.127 \pm 0.007$ & $\mathrm{Nm}$ & $0.143 \pm 0.007^{\#}$ & $0.143 \pm 0.008$ & $0.131 \pm 0.012^{\#}$ & $\mathrm{Nm}$ & 0.007 \\
\hline \multicolumn{9}{|c|}{$\mathrm{CRP}, \mathrm{mg} / \mathrm{L}$} \\
\hline Placebo & $1.9 \pm 0.7$ & $1.1 \pm 0.1$ & $1.3 \pm 0.3$ & $2.0 \pm 0.7$ & $1.6 \pm 0.7$ & $1.2 \pm 0.2$ & $1.6 \pm 0.4$ & \\
\hline ATP & $1.4 \pm 0.4$ & $1.1 \pm 0.1$ & $1.2 \pm 0.2$ & $1.9 \pm 0.6$ & $1.7 \pm 0.6$ & $1.1 \pm 0.1$ & $1.2 \pm 0.2$ & 0.99 \\
\hline \multicolumn{9}{|c|}{ Cortisol, $\mu \mathrm{g} / \mathrm{dL}$} \\
\hline Placebo & $19.7 \pm 1.1$ & $20.8 \pm 1.3$ & $19.0 \pm 1.2$ & $19.2 \pm 0.4$ & $22.0 \pm 0.4$ & $23.6 \pm 0.3$ & $20.3 \pm 0.6$ & \\
\hline ATP & $20.9 \pm 1.2$ & $20.5 \pm 1.3$ & $18.4 \pm 1.4$ & $19.0 \pm 0.4$ & $21.5 \pm 0.4$ & $22.6 \pm 0.2$ & $19.7 \pm 0.6$ & 0.86 \\
\hline \multicolumn{9}{|c|}{ Free Testosterone, ng/dL } \\
\hline Placebo & $103 \pm 13$ & $112 \pm 10$ & $119 \pm 6$ & $111 \pm 9$ & $98 \pm 6$ & $100 \pm 9$ & $113 \pm 12$ & \\
\hline ATP & $112 \pm 13$ & $114 \pm 9$ & $118 \pm 6$ & $117 \pm 11$ & $108 \pm 7$ & $110 \pm 10$ & $125 \pm 13$ & 0.93 \\
\hline \multicolumn{9}{|c|}{ Total Testosterone, ng/dL } \\
\hline Placebo & $591 \pm 73$ & $620 \pm 58$ & $625 \pm 55$ & $585 \pm 58$ & $551 \pm 46$ & $536 \pm 88$ & $605 \pm 72$ & \\
\hline ATP & $660 \pm 67$ & $645 \pm 54$ & $695 \pm 60$ & $645 \pm 60$ & $621 \pm 49$ & $592 \pm 84$ & $673 \pm 69$ & 0.83 \\
\hline \multicolumn{9}{|l|}{$\mathrm{PRS}^{\mathrm{d}}$} \\
\hline Placebo & $9.1 \pm 0.3$ & $4.7 \pm 0.4$ & $7.0 \pm 0.3$ & $7.6 \pm 0.2$ & $4.8 \pm 0.3$ & $4.4 \pm 0.3$ & $7.6 \pm 0.2$ & \\
\hline ATP & $9.6 \pm 0.2$ & $4.9 \pm 0.4$ & $7.5 \pm 0.3$ & $8.2 \pm 0.3$ & $5.5 \pm 0.4$ & $5.5 \pm 0.4$ & $8.6 \pm 0.4$ & 0.61 \\
\hline
\end{tabular}

${ }^{a}$ Mean \pm SEM for $n=11$ ATP (400 mg ATP/d taken 30 min prior to exercise) and $n=10$ placebo supplemented participants.

${ }^{b}$ Probability of treatment by time difference between the placebo and the ATP treatments over the 12-week study. The mixed model in SAS ${ }^{\circledR}$ was used with the main effects of treatment, week and treatment by week, with the value for week 0 used as a covariate. The mixed model in SAS ${ }^{\circledR}$ was also used for $3 \mathrm{MH}: \mathrm{Cr}$ however, $3 \mathrm{MH}: \mathrm{Cr}$ was measured only at weeks $0,1,8,9$, and 10 . The model used these values with week 0 as a covariate for determining the overall treatment by time effect for $3 \mathrm{MH}: \mathrm{Cr}$.

cNot measured.

${ }^{\mathrm{d}}$ Perceived recovery score is rated on the participants feeling of recovery from the last workout on a scale of $0-10.0-2$ indicates poor recovery and an anticipated decline in performance; 4-6 indicates low to moderate recovery and expected similar performance; and 8-10 indicates highly perceived recovery and expected increases in performance.

\#Significantly different than corresponding placebo, $t$-test $(p<0.05)$.

supplemental ATP on physiological responses that would improve long-term muscular performance. For example, Jordan et al. [13] demonstrated that 14 days of orally ingested ATP positively influenced exercise performance. Specifically, they demonstrated that $225 \mathrm{mg}$ per day of ATP for 14 days resulted in within-group increases in setone total repetitions performed and bench press total training volume. More recently, Rathmacher et al. [14] found that $400 \mathrm{mg}$ of supplemental ATP per day for 15 days was effective in improving set-two leg muscle minimum peak torque and tended to decrease set-three leg muscle fatigue during three successive sets of knee extension exercises. Interestingly, the changes observed during the aforementioned studies occurred despite final ATP supplementation being provided to test participants 3 [13] and 12 hours [14] prior to post-testing data collection.
The prolonged lag time between supplementation and testing may explain the inconclusive, albeit withingroup significant effects observed by Jordan et al. [13] and the somewhat minimal but between group effects on torque and fatigue observed by Rathmacher et al. [14] Furthermore, since the $400 \mathrm{mg}$ per day dose used by Rathmacher et al. [14] was divided into two doses throughout the day, the effective pre-exercise testing dose consumed prior to the 12-hour supplement withdrawal would have only been $200 \mathrm{mg}$ of ATP.

The current investigation employed once per day supplementation of a $400 \mathrm{mg}$ dose of ATP where on training and testing days participants consumed the supplement 30 minutes prior to exercise. We found the ATP supplementation resulted in strength increases for the squat and deadlift of $12.9 \%$ and $16.4 \%$, respectively, when compared 
Table 4 Blood chemistry values

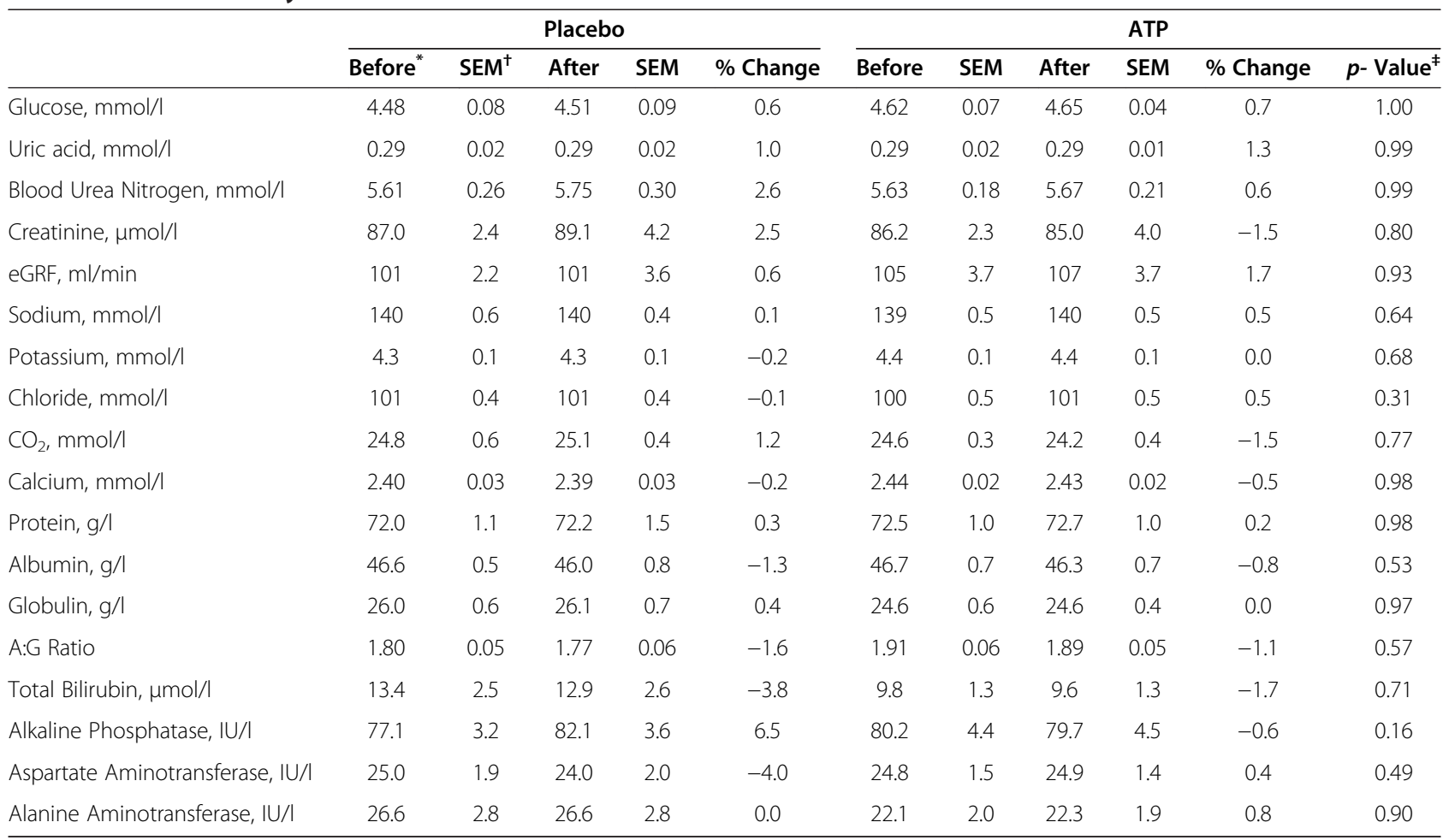

Values before and after 12 weeks of either placebo or ATP ( $400 \mathrm{mg} / \mathrm{d})$ administration.

${ }^{\dagger}$ Standard error of the mean.

${ }^{\ddagger} p$ - value for Trt*time effect, the model included data from weeks 4 and 8 (not shown).

to $\mathrm{RT}$ alone in the placebo ( $4.4 \%$ and $8.5 \%)$. In comparison to the two former studies, it is likely that the more robust changes observed within the current investigation are the result of total ATP dose and dose timing relative to testing procedures.

With regard to muscle power, we found that vertical jump peak power was more responsive to ATP supplementation $(+15.3 \%)$ as compared to placebo $(+11.5 \%)$. Rathmacher et al. [14] have speculated that supplemental ATP may provide cumulative benefits in strenuous, repetitive, and exhaustive exercise activities, which could lead to improvements in muscle responses. However, we have to point out that the mechanisms that ATP mediates changes in skeletal muscle performance are still under investigation and need to be fully elucidated. It can be speculated that these functional changes are a factor of small, transient increases in extracellular ATP and its metabolites. For example, Sandona et al. [25] presented evidence that $[\mathrm{ATP}]_{\mathrm{ex}}$ increases skeletal muscle $\mathrm{Ca}^{2+}$ influx and the release of $\mathrm{Ca}^{2+}$ from the sarcoplasmic reticulum, thereby affecting muscle contractile properties. Specifically, increasing skeletal muscle $\mathrm{Ca}^{2+}$ influx and intracellular concentrations have been shown to significantly increase both the total number of thin filaments binding and the speed at which the filaments slide [26]. These aforementioned findings provide a clue as to how the ATP supplementation might modulate the increases in muscle strength and power. However, these speculations must be eventually validated with direct research.

\section{The effects of ATP on skeletal muscle mass and changes in lean body mass}

To our knowledge, this study represents the first formal investigation of the effects of oral ATP supplementation on lean body mass and muscle thickness following a chronic RT program. Our results indicated greater increases in LBM and muscle thickness in response to oral ATP versus placebo. We can speculate on a number of possible reasons for the ergogenic effects of ATP on muscle mass we have observed. In addition to ATP's capacity to buffer fatigue during repeated high volume sets and increase total training volume, the supplement may increase skeletal muscle blood flow, thereby enhancing muscle $\mathrm{O}_{2}$ recovery. This is critical as muscle deoxygenation is associated with decreased performance under repeated high intensity contractions [27]. Specifically, extracellular ATP directly promotes the increased synthesis and release of nitric oxide (NO) and prostacyclin $\left(\mathrm{PGl}_{2}\right)$ within skeletal muscle and therefore directly affects tissue vasodilation and blood flow [28]. This is supported by research suggesting increased vasodilation 
Table 5 Blood hematology values

\begin{tabular}{|c|c|c|c|c|c|c|c|c|c|c|c|}
\hline & \multicolumn{5}{|c|}{ Placebo } & \multicolumn{6}{|c|}{ ATP } \\
\hline & Before $^{*}$ & $\mathrm{SEM}^{\dagger}$ & After & SEM & $\overline{\% \text { Change }}$ & Before & SEM & After & SEM & \% Change & $p$ Value $^{\ddagger}$ \\
\hline $\mathrm{WBC}, \times 10^{9} / \mathrm{I}$ & 5.93 & 0.38 & 5.98 & 0.34 & 0.8 & 6.08 & 0.24 & 6.07 & 0.22 & -0.1 & 0.67 \\
\hline $\mathrm{RBC}, \times 10^{12} / \mathrm{I}$ & 5.16 & 0.10 & 5.19 & 0.09 & 0.7 & 5.13 & 0.04 & 5.07 & 0.05 & -1.1 & 0.30 \\
\hline Hemoglobin, g/l & 156 & 2.8 & 155 & 2.4 & -1.0 & 157 & 2.2 & 156 & 2.0 & -0.8 & 0.97 \\
\hline Hematocrit, I/I & 0.46 & 0.01 & 0.46 & 0.01 & 0.0 & 0.47 & 0.01 & 0.46 & 0.01 & -1.8 & 0.54 \\
\hline$M C V, \mu m^{3}$ & 89.7 & 0.8 & 89.3 & 1.0 & -0.5 & 90.6 & 0.8 & 89.7 & 0.8 & -1.0 & 0.94 \\
\hline MCH, pg/cell & 30.5 & 0.40 & 30.2 & 0.33 & -1.1 & 30.4 & 0.32 & 30.5 & 0.44 & 0.3 & 0.42 \\
\hline $\mathrm{MCHC}, \mathrm{g} / \mathrm{l}$ & 339 & 3.1 & 336 & 2.5 & -0.9 & 334 & 3.9 & 340 & 4.4 & 2.1 & 0.16 \\
\hline $\mathrm{RDW}, \%$ & 13.4 & 0.1 & 13.4 & 0.1 & 0.0 & 13.1 & 0.1 & 13.2 & 0.1 & 0.8 & 0.62 \\
\hline Platelets, $\times 10^{9} / \mid$ & 247 & 9.0 & 236 & 9.1 & -4.2 & 248 & 9.5 & 217 & 5.1 & -12.5 & 0.64 \\
\hline Neutrophils,\% & 46.5 & 3.1 & 47.4 & 2.5 & 1.9 & 47.6 & 3.0 & 47.8 & 2.0 & 0.5 & 0.80 \\
\hline Lymphocytes,\% & 39.6 & 3.2 & 38.4 & 2.6 & -3.0 & 39.0 & 2.8 & 38.8 & 1.7 & -0.6 & 0.74 \\
\hline Monocytes, $\%$ & 10.3 & 0.6 & 9.4 & 0.7 & -8.7 & 9.3 & 0.5 & 9.6 & 0.6 & 3.0 & 0.57 \\
\hline Eosinophils,\% & 3.6 & 0.5 & 3.6 & 0.5 & 0.0 & 3.4 & 0.4 & 3.4 & 0.4 & 0.0 & 0.98 \\
\hline Basophils,\% & 0.6 & 0.2 & 0.7 & 0.2 & 16.7 & 0.4 & 0.2 & 0.4 & 0.2 & -0.3 & 0.83 \\
\hline Neutrophils, $\times 10^{9} / /$ & 3.2 & 0.6 & 3.3 & 0.5 & 2.5 & 3.0 & 0.3 & 2.9 & 0.2 & -2.3 & 0.27 \\
\hline Lymphocytes, $\times 10^{9} /$ & 2.4 & 0.2 & 2.5 & 0.3 & 4.1 & 2.4 & 0.2 & 2.4 & 0.1 & 2.9 & 0.99 \\
\hline Monocytes, $\times 10^{9} / \mathrm{I}$ & 0.67 & 0.08 & 0.62 & 0.08 & -7.5 & 0.82 & 0.20 & 0.61 & 0.05 & -25.6 & 0.62 \\
\hline Eosinophils, $\times 10^{9} / /$ & 0.21 & 0.02 & 0.21 & 0.02 & 0.0 & 0.21 & 0.03 & 0.22 & 0.03 & 4.4 & 0.32 \\
\hline Basophils, $\times 10^{9} / \mathrm{I}$ & 0.02 & 0.01 & 0.01 & 0.01 & -50.0 & 0.00 & 0.00 & 0.00 & 0.00 & 0.0 & 0.41 \\
\hline
\end{tabular}

"Values Before and After 12 weeks of either placebo or ATP (400 mg/d) administration.

${ }^{\dagger}$ Standard error of the mean.

${ }^{\ddagger} P$ value for Trt ${ }^{*}$ time effect, the model included data from weeks 4 and 8 (not shown).

and blood flow in response to intra-arterial infusion [29] and exogenous administration of ATP. It can be speculated that these changes in blood flow may lead to an increased substrate pool for skeletal muscle by virtue of increased glucose and $\mathrm{O}_{2}$ uptake [9]. If this is the case the resulting outcome would be an improved recovery response via a greater energetic environment for anabolic processes capable of supporting exercise-induced changes in LBM and hypertrophy. However, these are currently only suggested possible mechanisms and need to be verified in future research.

\section{The effects of ATP on recovery from high intensity training}

Overtraining and overreaching are two of the most complicated occurrences in sport. The primary tools utilized to detect overtraining and overreaching include changes in serum indices of skeletal muscle damage [30], anabolic and catabolic hormone status [31], perceived recovery [20], and muscle protein breakdown. However, the consensus seems to dictate that the number one indicator of overreaching and overtraining are short- and long-term decrements in performance, respectively [32]. The cause of overreaching appears to be an imbalance between training stimulus and recovery. If the stimulus exceeds the athlete's adaptive capacity, decrements in performance will result; ultimately taking weeks (overreaching) to months (overtraining) to fully recover. For ethical reasons, a great deal of research in strength and conditioning has centered on overreaching protocols versus overtraining [32].

The present study attempted to overreach participants through increasing training frequency and volume. Our results indicated that the overreaching cycle was able to decrease muscle power and strength, and overreaching increased protein breakdown. However, these effects were blunted in the ATP group. It is interesting to note that protein breakdown was not blunted during phase 1 (weeks 1-8) by ATP supplementation. In fact, although protein breakdown in the ATP supplemented group was not significantly different than baseline, it appeared slightly higher at week 8 relative to the control. We can speculate that under normal conditions of training, when glycogen levels are likely adequate those participants supplementing with ATP were able to maintain higher intensities, which would result in higher rates of protein breakdown. However, when exposed to greater training frequencies, glycogen levels are likely to be depleted, thus preventing higher intensities from being performed. As such ATP supplementation is able to blunt a rise in protein breakdown relative to a placebo 
group. Thus, it appears that oral ATP may be able to increase fatigue resistance during a two-week intensive, high-volume overreaching protocol. Following a two-week taper in which volume was reduced, the placebo group regained their baseline performance while the ATP group experienced increases in both muscle strength and power. These results indicate that a typical overreaching stimulus overwhelms recovery capabilities in a non-supplemented state. However, the ability for ATP to speed recovery may provide athletes with a novel method to promote positive training adaptations.

While at first look these results may appear to be only pertinent to athletic performance, it is important to understand that several non-sport activities place people at risk for deterioration of performance in life threatening situations where performance is critical. A primary example includes combat athletes / military personal who are often times placed in extreme overreaching and overtraining environments that may take months to recover from [33]. These are often times highly conditioned individuals whose lives and mission may depend on the prevention of decay in strength, and power.

\section{Effects of chronic ATP supplementation on safety parameters}

The results of this study suggest that $400 \mathrm{mg}$ of oral ATP administered daily had no effect on hemoglobin, white blood cells, blood glucose, liver, or kidney function. These results are in agreement with Coolen et al. [34] who reported that up to $5000 \mathrm{mg}$ per day, during 28 days, of oral ATP resulted in no significant changes in any blood or urine measured safety parameters. Therefore, it appears likely that doses of $400 \mathrm{mg} /$ day of oral ATP for up to 12 weeks can be considered safe and presented no clinically relevant adverse effects.

\section{Bioavailability of oral ATP supplementation}

Recently, Coolen et al. questioned the bioavailability of oral ATP [2]. However, the biological pool where ATP is measured will determine the results of bioavailability analysis. If sampled in venous portal blood, oral ATP is indeed bioavailable [13]. Coolen et al. did not analyse venous portal blood. If sampling systemic plasma or whole blood, oral ATP will have very low bioavailability even in very high doses. Paradoxically, Kichenin \& Seman observed that repeated administration of oral ATP led to progressive diminution of plasma ATP [12]. Nevertheless, their study demonstrated that oral ATP supplementation can indeed produce biological responses. Over all, due to the nature of the ATP molecule, its bioavailability is quite difficult to determine. While our results suggest that oral ATP supplementation can significantly impact athletic performance, skeletal muscle hypertrophy and recovery, the current study did not utilize methodologies to investigate the potential mechanism(s) for the ergogenic effects we observed.

\section{Limitations}

This study, similar to others, has limitations. This study included all male subjects; therefore, future research will need to expand our study design to a female population. We also acknowledge that that these results occurred with a highly trained population under extreme training conditions. Chronic studies will need to be conducted in untrained individuals under normal training loads. Finally, we did not assess changes in muscular $\mathrm{O}_{2}$ concentrations or changes in intramuscular ATP. We suggest that future researchers conduct these studies using near infrared spectroscopy to access intramuscular $\mathrm{O}_{2}$ concentrations and magnetic resonance spectroscopy to assess any changes in ATP charge. Without these measures, caution should be taken when interpreting the actual mechanisms of action of the supplement.

\section{Conclusions}

The collective findings of our current study suggest that oral supplementation with ATP in combination with high intensity, periodized RT, increases muscle mass, strength, and power compared with a placebo-matched control. Moreover, when faced with greater training frequencies, oral ATP may prevent typical declines in performance that are characteristic of overreaching. Future research should seek to elucidate the underlying mechanisms through which ATP operates to promote improvements in training adaptations.

\section{Abbreviations}

ATP: Adenosine-5'-triphosphate; Ck: Creatine kinase; cGMP: Current Good Manufacturing Practices; DXA: Dual-energy x-ray absorptiometry; LBM: Lean body mass; PP: Peak power; $\mathrm{PGI}_{2}$ : Prostacyclin; RT: Resistance training.

\section{Competing interests}

JMW, JMJ, RPL, MDR, EOD, SMCW and AHM declare no competing interests. $J R$, JF, and SB are employed by Metabolic Technologies, Inc. which engages in business trade with TSI (USA), Inc. CL was a consultant of TSI, Inc.

\section{Authors' contribution}

$J M W, R P L, J M J$ were involved in study design, training subjects, biochemical analysis of blood, data interpretation, and manuscript preparation. JAR, SMB, and JCF were involved in study design, supplement preparation, analysis of 3-MH, statistical analysis, and manuscript preparation. MDR, CML, AHM, and EOD were critical for study design, and manuscript preparation. SMCW served as the study's sports dietitian and also assisted in study design. All authors read and approved the final manuscript.

\section{Acknowledgements}

This research was funded in part through a grant from TSI (USA), Inc., Missoula, MT to JMW. TSI (USA), Inc. also provided the Peak ATP® and placebo supplements used in the study.

\section{Author details}

${ }^{1}$ Department of Health Sciences and Human Performance, The University of Tampa, Tampa FL, USA. ${ }^{2}$ Department of Biomedical Sciences, College of Veterinary Medicine, University of Missouri, Columbia, MO, USA. ${ }^{3}$ AP Nutrition, LLC, Missoula MT, USA. ${ }^{4}$ Metabolia Oulu, Oulu, Finland. ${ }^{5}$ Metabolic 
Technologies Inc., lowa State University Research Park, Ames, IA, USA. ${ }^{6}$ Laboratory of Neuromuscular Adaptations to Strength Training, School of Physical Education and Sport, University of São Paulo, São Paulo, Brazil. ${ }^{7}$ Department of Nutrition, IMG Academy, Bradenton, FL, USA. ${ }^{8}$ Department of Animal Science, lowa State University, Ames, IA, USA.

Received: 19 June 2013 Accepted: 13 September 2013 Published: 22 September 2013

\section{References}

1. Kushmerick MJ, Conley KE: Energetics of muscle contraction: the whole is less than the sum of its parts. Biochem Soc Trans 2002, 30(2):227-231.

2. Burnstock G, Knight GE, Greig AV: Purinergic signaling in healthy and diseased skin. J Invest Dermatol 2012, 132(3 Pt 1):526-546.

3. Agteresch HJ, Dagnelie PC, van den Berg JW, Wilson JH: Adenosine triphosphate: established and potential clinical applications. Drugs 1999 58(2):211-232.

4. Sawynok J, Sweeney MI: The role of purines in nociception. Neuroscience 1989, 32(3):557-569.

5. Khakh BS, Henderson G: ATP receptor-mediated enhancement of fast excitatory neurotransmitter release in the brain. Mol Pharmacol 1998, 54(2):372-378

6. Gorman MW, Feigl EO, Buffington CW: Human plasma ATP concentration Clin Chem 2007, 53(2):318-325.

7. Hochachka PW, Bianconcini MS, Parkhouse WS, Dobson GP: On the role of actomyosin ATPases in regulation of ATP turnover rates during intense exercise. Proc Natl Acad Sci U S A 1991, 88(13):5764-5768.

8. Mortensen SP, Thaning P, Nyberg M, Saltin B, Hellsten Y: Local release of ATP into the arterial inflow and venous drainage of human skeletal muscle: insight from ATP determination with the intravascular microdialysis technique. J Physio/ 2011, 589(Pt 7):1847-1857.

9. Ellis CG, Milkovich S, Goldman D: What is the efficiency of ATP signaling from erythrocytes to regulate distribution of $\mathrm{O}(2)$ supply within the microvasculature? Microcirculation 2012, 19(5):440-450

10. Sprague RS, Bowles EA, Achilleus D, Ellsworth ML: Erythrocytes as controllers of perfusion distribution in the microvasculature of skeletal muscle. Acta Physiol 2011, 202(3):285-292.

11. Trautmann A: Extracellular ATP, in the immune system: more than just a "danger signal". Sci Signal 2009, 2(56):pe6.

12. Kichenin K, Seman M: Chronic oral administration of ATP modulates nucleoside transport and purine metabolism in rats. J Pharmacol Exp Ther 2000, 294(1):126-133.

13. Jordan AN, Jurca R, Abraham EH, Salikhova A, Mann JK, Morss GM, Church TS, Lucia A, Earnest CP: Effects of oral ATP supplementation on anaerobic power and muscular strength. Med Sci Sports Exerc 2004, 36(6):983-990

14. Rathmacher JA, Fuller JC Jr, Baier SM, Abumrad NN, Angus HF, Sharp RL: Adenosine-5'-triphosphate (ATP) supplementation improves low peak muscle torque and torque fatigue during repeated high intensity exercise sets. Journal of the International Society of Sports Nutrition 2012 9(1):48.

15. Kraemer WJ, Hatfield DL, Volek JS, Fragala MS, Vingren $J$, Anderson JM, Spiering BA, Thomas GA, Ho JY, Quann EE, Izquierdo M, Hakkinen K, Maresh CM: Effects of amino acids supplement on physiological adaptations to resistance training. Med Sci Sports Exerc 2009, 41(5):1111-1121.

16. Gilbert $G$, Lees A: Changes in the force development characteristics of muscle following repeated maximum force and power exercise. Ergonomics 2005, 48(11-14):1576-1584.

17. Weir JP: Quantifying test-retest reliability using the intraclass correlation coefficient and the SEM. J Strength Cond Res 2005, 19(1):231-240.

18. Smith JC, Fry AC, Weiss LW, Li Y, Kinzey SJ: The effects of high-intensity exercise on a 10-second sprint cycle test. J Strength Cond Res 2001, 15(3):344-348

19. Lowery RP, Duncan NM, Loenneke JP, Sikorski EM, Naimo MA, Brown LE, Wilson FG, Wilson JM: The effects of potentiating stimuli intensity under varying rest periods on vertical jump performance and power. J Strength Cond Res 2012, 26(12):3320-3325.

20. Ackel-D'Elia C, Vancini RL, Castelo A, Nouailhetas VL, Silva AC: Absence of the predisposing factors and signs and symptoms usually associated with overreaching and overtraining in physical fitness centers. Clinics (Sao Paulo) 2010, 65(11):1161-1166.
21. Robbins DW, Docherty D: Effect of loading on enhancement of power performance over three consecutive trials. J Strength Cond Res 2005, 19(4):898-902

22. Wilson JM, Duncan NM, Marin PJ, Brown LE, Loenneke JP, Wilson SM, Jo E, Lowery RP, Ugrinowitsch C: Meta-Analysis of Post Activation Potentiation and Power: Effects of Conditioning Activity, Volume, Gender, Rest Periods, and Training Status. J Strength Cond Res 2013, 27(3):854-859.

23. Cormie P, McGuigan MR, Newton RU: Developing maximal neuromuscular power: Part 1-biological basis of maximal power production. Sports Med 2011, 41(1):17-38

24. Cormie P, McGuigan MR, Newton RU: Developing maximal neuromuscular power: part 2 - training considerations for improving maximal power production. Sports Med 2011, 41(2):125-146.

25. Sandona D, Danieli-Betto D, Germinario E, Biral D, Martinello T, Lioy A, Tarricone E, Gastaldello S, Betto R: The T-tubule membrane ATP-operated P2X4 receptor influences contractility of skeletal muscle. FASEB J 2005, 19(9):1184-1186.

26. Homsher E, Kim B, Bobkova A, Tobacman LS: Calcium regulation of thin filament movement in an in vitro motility assay. Biophys J 1996, 70(4):1881-1892

27. Buchheit M, Cormie P, Abbiss CR, Ahmaidi S, Nosaka KK, Laursen PB: Muscle deoxygenation during repeated sprint running: Effect of active vs. passive recovery. Int J Sports Med 2009, 30(6):418-425.

28. Nyberg M, Mortensen SP, Thaning P, Saltin B, Hellsten Y: Interstitial and plasma adenosine stimulate nitric oxide and prostacyclin formation in human skeletal muscle. Hypertension 2010, 56(6):1102-1108.

29. Gonzalez-Alonso J, Mortensen SP, Jeppesen TD, Ali L, Barker H, Damsgaard R, Secher NH, Dawson EA, Dufour SP: Haemodynamic responses to exercise, ATP infusion and thigh compression in humans: insight into the role of muscle mechanisms on cardiovascular function. J Physiol 2008, 586(9):2405-2417.

30. Xiao W, Chen P, Dong J: Effects of overtraining on skeletal muscle growth and gene expression. Int J Sports Med 2012, 33(10):846-853.

31. Tanskanen MM, Kyrolainen H, Uusitalo AL, Huovinen J, Nissila J, Kinnunen $H_{\text {, }}$ Atalay M, Hakkinen K: Serum sex hormone-binding globulin and cortisol concentrations are associated with overreaching during strenuous military training. J Strength Cond Res 2011, 25(3):787-797.

32. Halson $\mathrm{SL}$, Jeukendrup AE: Does overtraining exist? An analysis of overreaching and overtraining research. Sports Med 2004, 34(14):967-981.

33. Chicharro JL, Lopez-Mojares LM, Lucia A, Perez M, Alvarez J, Labanda P, Calvo F, Vaquero AF: Overtraining parameters in special military units. Aviat Space Environ Med 1998, 69(6):562-568.

34. Coolen EJ, Arts IC, Bekers O, Vervaet C, Bast A, Dagnelie PC: Oral bioavailability of ATP after prolonged administration. Br J Nutr 2011 105(3):357-366.

\section{doi:10.1186/1743-7075-10-57}

Cite this article as: Wilson et al: Effects of oral adenosine-5'-triphosphate supplementation on athletic performance, skeletal muscle hypertrophy and recovery in resistance-trained men. Nutrition \& Metabolism 2013 10:57.

\section{Submit your next manuscript to BioMed Central and take full advantage of:}

- Convenient online submission

- Thorough peer review

- No space constraints or color figure charges

- Immediate publication on acceptance

- Inclusion in PubMed, CAS, Scopus and Google Scholar

- Research which is freely available for redistribution 